DOI: $10.14451 / 2.126 .67$

\title{
ОЦЕНКА ЭФФЕКТИВНОСТИ ИНФОРМАЦИОННЫХ СИСТЕМ УПРАВЛЕНИЯ ПРЕДПРИЯТИЕМ В УСЛОВИЯХ ИНФОРМАЦИОННОЙ ГЛОБАЛИЗАЦИИ
}

\author{
(c) 2018 Жук Марина Алексеевна \\ доктор экономических наук, доцент \\ заведующий кафедрой прикладной информатики в экономике и управлении \\ Оренбургский государственный университет \\ 460018, г. Оренбург, пр. Победы, д. 13 \\ E-mail: eng_m@inbox.ru
}

В исследовании рассматривается проблема оценки эффективности корпоративных информационных систем как активных элементов экономико-информационного пространства предприятий, реализующих виртуальное экономическое взаимодействие предприятия с другими хозяйствующими субъектами в глобальной информационной инфраструктуре.

Ключевые слова: информационная система, глобальная инфраструктура, экономико-информационное пространство предприятия, эффективность информационных систем управления предприятием, критерий эффективности информационной системы.

В настоящее время можно говорить об устойчивом развитии информационного общества, затрагивающего и трансформирующего все социально-экономические процессы. Трансформация экономического взаимодействия субъектов в означенных условиях наиболее выражена в формировании глобальной информационной инфраструктуры как пространства экономического взаимодействия хозяйствующих субъектов различной природы. В настоящее время уровень вхождения нашего государства в глобальную информационную инфраструктуру накладывает совершенно новые, технологически обоснованные требования к оценке эффективности функционирования информационных систем на всех уровнях управления. Более того, в современных условиях уровень интеграции любого предприятия в глобальную информационную инфраструктуру во многом определяет уровень его конкурентоспособности.

В условиях развитого информационного общества многократно возрастает интенсивность обмена информацией между предприятием (как системой) и элементами микро и макро внешней среды по отношению к предприятию. К микросреде могут быть отнесены предприятия партнеры, поставщики и собственно клиенты (потребители производимого предприятием продукта), макросреда представлена органами государственной власти, крупными предприятиями и холдингами, определяющими промышленную политику в означенном сегменте. Кроме этого, происходит интенсификация информа- ционных потоков внутри предприятия, это касается и вертикальных потоков (нисходящих и восходящих) и потоков горизонтальных, касающихся обмена информацией между подразделениями и сотрудниками. Более того, усиление интенсивности горизонтальных потоков постепенно привело к трансформации организационных структур - явилось причиной возрастания популярности проектных и матричных организационных структур, которые позволяют повысить эффективность работы предприятия в целом, разрабатывать и достаточно оперативно внедрять инновационные проекты, изыскивать внутренние ресурсы для развития, сокращать уровни управления. Безусловно, подобные организационные структуры являются более оптимальными, с точки зрения парадигмы ресурсосберегающей организации.

В последнее десятилетие в исследованиях, посвященных проблемам управления корпоративными информационными системами, широко используется понятие «экономико-информационное пространство предприятия» $[5,6]$. Оно определяется как некоторая интегрирующая форма совокупности информационных подсистем, как самого предприятия, так и информационных систем, взаимодействующих с ним посредством виртуальной среды хозяйствующих субъектов. Каждая информационная подсистема, функционирующая в информационном пространстве предприятия, представляется как некоторый объект, обладающий информационным ресурсом, задействованном в процессе эко- 
номического взаимодействия субъектов. В связи с вышесказанным приведенное понятие может быть уточнено как экономико-информационное пространство предприятия [5], представляющее собой часть глобальной информационной инфраструктуры и являющееся платформой экономического взаимодействия предприятий в виртуальной среде.

Рассматривая предприятие как активную экономико-информационную подсистему глобального информационного пространства, особую важность приобретают вопросы эффективности корпоративных информационных систем предприятия, как структур, аккумулирующих информационный ресурс. При наличии достаточного количества исследований, посвященных процессам разработки и внедрения современных корпоративных информационных систем на предприятии, методики оценки эффективности информационных систем значительно устарели, не только в практических, но даже в терминологических аспектах. Рассмотрим проблему формирования терминологического аппарата, используемого при оценке экономической эффективности информационных систем более подробно. Эффективность как понятие формировалось на протяжении длительного периода времени, последовательно обогащаясь и расширяя сферы применения. Можно говорить о ключевом значении данного понятия для экономики и управления, но при этом, оно также является ключевым в системном анализе и кибернетике. В работах отечественных ученых, например [7], понятие эффективности обоснованно рассматривается в широком и узком смыслах.

Говоря о широком смысле понятия эффективности, рассматривается некоторая комплексная характеристика практической полезности информационной системы. Подобная характеристика может быть рассмотрена как некоторый интегральный эффект, в свою очередь понятие полезности рассматривается как совокупность практических результатов. Тогда при определении интегрального эффекта анализируется соответствие полученных результатов целям и ресурсам предприятия.

Принято считать, что анализ эффективности в широком смысле может быть рассмотрен как три базовые задачи [2] системного анализа:

- исследование эффективности процессов функционирования системы в реальном объекте приложения (предприятие);
- выбор наилучшего варианта системы из имеющегося множества вариантов, выбранный вариант должен является оптимальным по эффективности;

- структурный синтез системы, удовлетворяющей всем наборам требований оптимальности.

Для определения понятия эффективность в узком смысле необходимо декомпозировать вышеприведенное понятие, руководствуясь принципом разделения по направлениям: экономическая, техническая, экологическая и т.п.

Говоря об экономической эффективности, можно рассматривать ее как ключевую форму проявления эффективности сообщества социальных и хозяйствующих субъектов. По сути, понятие экономической эффективности универсально в своем применении для оценки функционирования большого класса систем, так в ее основе лежит принцип сравнительной оценки получаемого результата и ресурсов, затраченных на его получение в рамках установленных временных интервалов, оценка производится в единой стоимостной мере. Не менее важным направлением определения «узкой» эффективности является определение эффективности управления. При этом следует рассматривать данное понятие и как самостоятельный частный вид эффективности, и в приложении к большим техническим системам, включающим в свой состав автоматизированные системы управления.

Корпоративные информационные системы могут быть определены и как сложные технические системы, и как системы управления, а с учетом того, что в процессе их функционирования обрабатываются потоки экономической информации - их можно отнести и к классу экономических систем. В связи с этим проблема определения эффективности корпоративных информационных систем (КИС) включает в себя аспекты технической, управленческой и экономической природы. Рассмотрим определение технической системы, приведенное в [8]: техническая система - это материальная, неживая функционально-ориентированная система, которая входит в качестве элемента в надсистемную структуру и используется и/или развивается на основе мыслительных образов человека или сообщества.

Безусловно, информационные системы можно отнести к классу сложных систем, обладающих высокой степенью видового многооб- 
разия, это обуславливает наличие полисемии, которая влияет на понимание результатов работы информационных систем. Основной проблемой толкования понятия эффективности таких систем является снижение роли человеческого фактора, хотя без него само их существование теряет смысл. В этом аспекте наиболее обобщающим является определение, приведенное в [8] - «техническая система - искусственно созданная совокупность упорядоченных взаимодействующих элементов, обладающая синергетическим эффектом и созданная на основе моделей для удовлетворения определенных потребностей человека». В настоящее время информационные системы расширили рамки своей функциональности в области систем поддержки принятия решений, что обуславливает необходимость расширения определения этого понятия. В исследовании [4] это понятие трактуется как в широком, так и в узком смысле. Так, информационная система рассматривается как совокупность аппаратного, программного и организационного обеспечения, дополненная персоналом, нацеленным на удовлетворение информационных потребностей, заложенных в самой цели создания системы.

Проблема повышения эффективности информационной системы затрагивает вопросы ее устойчивого функционирования для поддержания работы пользовательских приложений, требования к которым заранее определены заказчиком, еще на стадии создания информационной системы. В связи с эти имеет смысл говорить об анализе состояний информационной системы на определенные моменты времени (текущий и будущие). Состояния информационной системы обуславливаются отраслевыми особенностями их применения, в частности - отраслевой принадлежностью предприятия для которого она является надстройкой аппарата управления. Каждое состояние подлежит комплексной оценке со стороны «заинтересованных лиц»- лиц, принимающих управленческие решения. Оценка состояний информационной системы как единого (неделимого) объекта исследования невозможна, в [7] рассматривается концепция оценки состояния информационной системы как совокупности сложных прикладных объектов. Таким образом, в узком смысле информационная система рассматривается с точки зрения много модельного принципа, используемого в системном анализе. Согласно этому принципу каждый прикладной объект может быть представлен совокупностью концептуальных моделей, отражающих аспекты реального объекта.

Обобщая широкий и узкий смысл понятия информационной системы можно определить ее как аппаратно-программный комплекс, устойчиво удовлетворяющий информационные потребности пользователей, дополненный подсистемой обработки о состояниях (и их изменениях) сложных прикладных объектов. При этом комплексная оценка состояния таких объектов рассматривается как эффективность информационной системы, которая в свою очередь может быть представлена набором частных критериев. Таким образом, актуальной является задача определения состава и структуры подобных критериев.

В [2] предлагается разбить критерии оценки эффективности информационной системы на три группы:

- критерии, характеризующие устойчивость информационной системы, как технического средства поддержки работы пользовательских приложений (производительность, помехоустойчивость и т.п.);

- критерии, характеризующие состояния сложных прикладных объектов с точки зрения их полезности в процессе принятия решения (ранжируемость состояний объекта относительно предпочтений лица, принимающего решения (ЛПР), степень адекватности выбранной модели предпочтений ценностным координатам ЛПР, уровни адекватности изменений каждого из прикладных объектов, изменению состояния информационной системы в целом, степень реализации управленческих компетенций посредством интеграции информационной системы в процесс принятия управленческих решений и т.п.);

- критерии, характеризующие степень интеллектуальности обработки информации об изменении уровней эффективности (изменении состояний) прикладных объектов, входящих в состав информационной системы: ранжируемость изменений состояния системы относительно координат ценностей ЛПР, уровень востребованности системы в управленческом процессе, уровень устойчивости системы попыткам манипулирования изменениями состояний объектов и т.п.).

Очевидно, что первую группу критериев необходимо дополнить экономическими критери- 
ями и механизмом интеграции всех показателей с целью получения комплексной оценки эффективности. Остальные группы критериев характеризуют уровень развития информационной системы на текущем этапе функционирования и уровень ее востребованности в процессах принятия управленческих решений. Их агрегация не имеет смысла, так как оценка осуществляется по каждой группе автономной.

Следует отметить тот факт, что использование традиционных методов системного анализа в процессе оценки разнородных показателей эффективности сложных систем современного уровня развития инфо-коммуникационных технологий неприемлемо, так как в данном случае необходима комплексная оценка. В данном случае необходимо учитывать все предпочтения (как индивидуальные, так и коллективные) всех заинтересованных лиц, так как речь идет об оценке динамики изменения уровня эффективности информационной системы. При этом в качестве заинтересованных лиц рассматриваются лица, принимающие решения, а их предпочтения - это интуитивные суждения эвристического характера, формирующиеся в процессе эксплуатации информационной системы.

Более того, можно говорить о наличии прямой связи между понятием эффективности информационной системы и совокупностью понятий, характеризующих конкурентоспособность информационной системы на рынке. При этом система предпочтений пользователей информационной системы может быть дополнена системой предпочтений участников рынка. В связи с этим возникает ряд актуальных вопросов, напрямую связанных с проблемой повышения эффективности информационных систем промышленных предприятий, к таковым можно отнести:

- формирование спроса на различные виды и модификации информационных систем предприятия;

- причины формирования той или иной системы предпочтений у лиц, принимающих решения в сфере разработки и внедрения информационных систем на предприятии;

- рациональное использование как уже имеющих, так и аккумулируемых на предприятии информационных ресурсов.

Таким образом, в условиях стремительного развития информационно-коммуникационных технологий эффективность информационных систем предприятия некорректно оценивать без учета динамики сегмента рынка, соответствующего отрасли предприятия, развивающегося в условиях информационной глобализации. В связи с этим - далее целесообразно рассматривать понятие эффективности информационной системы как части глобального экономико-информационного пространства [5].

Проблему развития глобальной информационной инфраструктуры можно рассматривать как «проблему обмена идеями, мнениями и знаниями в условиях территориальной разобщенности их носителей» [6], сама инфраструктура объединяет информационные пространства различной природы: территориально ограниченные (региональные и государственные), отраслевые, пространства социально-ориентированных сегментов. Во всех трех случаях можно говорить об экономической составляющей, поскольку в информационном пространстве любой природы осуществляется взаимодействие целеориентированных субъектов (или групп). На уровне предприятия процесс формирования глобальной информационной инфраструктуры должен обеспечить решение таких важных задач как [5]:

- систематизация информацию, обрабатываемой на предприятии, и организация единой базы информационных ресурсов, в состав которых входят: документы, оргструктура, сведения о персонале, проекты, бизнес-процессы и т.д.;

- обеспечение процесса управления знаниями (накопление, структуризация и распространение знаний);

- обеспечение для сотрудников единой точки доступа ко всем информационным ресурсам предприятия (принцип «единого окна»);

- автоматизация процессов административно-хозяйственной деятельности.

С целью интеграции информационных систем предприятий в глобальную структуру, в качестве обязательного требования вводится переход на уровень корпоративных систем типа В2В, ядром которых являются двухуровневые автоматизированные управленческие информационные системы. Концепция подобных систем включает два уровня: - микроуровень, который отражает информационное взаимодействие структурообразующих элементов предприятия (административные и производственно-технологические подразделения); - макроуровень, содержащий информацию о взаимодействии 
предприятия с внешней средой (клиенты, поставщики, другие предприятия и организации). Очевидно, что такие системы являются движущей силой реструктуризации и оптимизации бизнес-процессов для повышения эффективности производства и взаимодействия с поставщиками и клиентами. Типовыми подсистемами двухуровневых автоматизированных систем управления предприятием являются модули, обеспечивающие управление финансами, производством, складскими запасами, взаимоотношениями с поставщиками и клиентами, а также кадрами и делопроизводством.

По сути, подобные системы вписываются в идеологию корпоративных информационных систем. В свою очередь корпоративная идеология предполагает объединение хозяйствующих субъектов различных или одинаковых организационно-правовых форм и направления деятельности на временной или постоянной основе своих усилий для достижения совместных целей [5]. Объединение хозяйствующих субъектов в глобальной информационной инфраструктуре подразумевает более гибкие механизмы интеграции, так как все хозяйственные взаимодействия могут осуществляться дистанционно, без территориальных изменений.

\section{Библиографический список}

1. Белых А.А. Интеллектуальные технологии повышения эффективности информационных систем // Научный журнал КубГАУ, № 60(06), 2010 г._- http://ej.kubagro.ru/2010/06/pdf/35.pdf.

2. Белых А.А. Проблема повышения эффективности информационных систем в современных условиях // Научный журнал КубГАУ, № 58(04), 2010 г. - http://ej.kubagro.ru/2010/04/pdf/04.pdf.

3. Бурков В.Н., Новиков Д.А., Щепкина А.В. Механизмы управления экологоэкономическими системами / Под ред. Академика С.Н. Васильева. Москва. 2008. 244 с.

4. Бурков В.Н., Новиков Д.А. Теория активных систем: состояние и перспективы. Москва. 1999. 128 с.

5. Ковалевский В.П., Буреш О.В., Жук М.А., Калиева О.М. Аккумуляция знаний в информационном пространстве региона. Москва. Финансы и статистика. 2011. 352 с.

6. Калинина А.Э. Развитие информационного пространства региональной хозяйственной системы / А.Э. Калинина. Волгоград. 2012. С. 14-26.

7. Резников Б.А. Системный анализ и методы системотехники. Часть 1. Методология системных исследований. Моделирование сложных систем. Москва. 1990. 522 с

8. Рубин М.С. Этюды о законах развития техники. 2006 - http://www.temm.ru/ru/section.php?docId=3432

9. Nonaka I., Takeuchi I. The Knowledge-Creating Company // Oxford University Press: Oxford, 1995.189 p. 